\title{
Tauberian theorems for weighted mean statistical summability of double sequences of fuzzy numbers
}

\author{
Hemen Dutta and Jyotishmaan Gogoi
}

\begin{abstract}
We discuss Tauberian conditions under which the statistical convergence of double sequences of fuzzy numbers follows from the statistical convergence of their weighted means. We also prove some other results which are necessary to establish the main results.
\end{abstract}

\section{Introduction}

Since the introduction by Zadeh [27], the concept of fuzzy set theory and its applications have attracted the attention of many researchers from various branches of mathematics. Dubois and Prade [8] introduced the notion of fuzzy numbers and defined the basic operations of addition, subtraction, multiplication and division. Goetschel and Voxman gave a less restrictive definition of fuzzy numbers in [13]. Matloka [15] introduced the concepts of bounded and convergent sequences of fuzzy numbers and studied their properties.

In 1935, Zygmund [28] defined a new type of convergence known as almost convergence that formed the foundation of the concept of statistical convergence which was formally introduced by Fast [11] and reintroduced by Schoenberg [23] and also, independently by Buck [4]. Later this idea was associated with summability theory. Nuray and Savaş [18] extended the concept of statistical convergence to sequences of fuzzy numbers and showed that a sequence of fuzzy numbers is statistically convergent if and only if it is statistically Cauchy.

Recently there has been an increasing interest in summability methods of sequences of fuzzy numbers. In [24], Subrahmanyam defined the Cesàro

Received April 3, 2018.

2020 Mathematics Subject Classification. Primary 03E72; secondary 40A05, 40E05, $40 \mathrm{G} 15$.

Key words and phrases. Double sequences of fuzzy numbers, weighted mean statistical summability, Tauberian theorems.

https://doi.org/10.12697/ACUTM.2021.25.12 
summability method for sequences of fuzzy numbers and proved fuzzy analogues of some classical Tauberian theorems. For a detailed study and some results related to convergence of sequences of fuzzy numbers and Tauberian conditions, we refer to the papers $[1,3,5,9,10,14,19,26]$.

We now give some preliminary definitions and notations which are required in the later part of the paper.

Definition 1.1 (see $[8,13]$ ). A fuzzy number is a fuzzy set on the real axis, i.e., a mapping $u: \mathbb{R} \rightarrow[0,1]$ which satisfies the following four conditions:

(i) $u$ is normal, i.e., there exists an $x_{0} \in \mathbb{R}$ such that $u\left(x_{0}\right)=1$,

(ii) $u$ is fuzzy convex, i.e., $u[\lambda x+(1-\lambda) y] \geq \min \{u(x), v(y)\}$ for all $x, y \in \mathbb{R}$ and for all $\lambda \in[0,1]$,

(iii) $u$ is upper semi-continuous,

(iv) the set $[u]_{0}=\overline{\{x \in \mathbb{R}(x)>0\}}$ is compact, where $\overline{\{x \in \mathbb{R}(x)>0\}}$ denotes the closure of the set $\{x \in \mathbb{R}(x)>0\}$ in the usual topology of $\mathbb{R}$.

The set of all fuzzy numbers on $\mathbb{R}$ is denoted by $E^{1}$ and called the space of fuzzy numbers. The $\alpha$-level set $[u]_{\alpha}$ of $u \in E^{1}$ is defined by

$$
[u]_{\alpha}= \begin{cases}\{t \in \mathbb{R}: u(t) \geq \alpha\} & \text { if } 0<\alpha \leq 1, \\ \{t \in \mathbb{R}: u(t)>\alpha\} & \text { if } \alpha=0 .\end{cases}
$$

The set $[u]_{\alpha}$ is a closed, bounded, and non-empty interval for each $\alpha \in$ $[0,1]$, it is defined by $[u]_{\alpha}=\left[u^{-}(\alpha), u^{+}(\alpha)\right]$. The set $\mathbb{R}$ can be embedded in $E^{1}$, since each $r \in \mathbb{R}$ may be regarded as the fuzzy number

$$
\bar{r}(t)= \begin{cases}1 & \text { if } t=r \\ 0 & \text { if } t \neq r\end{cases}
$$

Definition 1.2 (see $[2,8]$ ). Let $u, v, w \in E^{1}$ and $k \in \mathbb{R}$. The addition, scalar multiplication, and product in $E^{1}$ are defined as follows:

$$
\begin{aligned}
& u+v=w \Leftrightarrow[w]_{\alpha}=[u]_{\alpha}+[v]_{\alpha} \quad(\alpha \in[0,1]), \\
& \left.k u=w \Leftrightarrow[w]_{\alpha}=k[u]_{\alpha} \quad(\alpha \in 0,1]\right), \\
& u v=w \Leftrightarrow[w]_{\alpha}=[u]_{\alpha}[v]_{\alpha} \quad(\alpha \in[0,1]) .
\end{aligned}
$$

Definition 1.3 (see [7]). Let $W$ be the set of all closed bounded intervals $A=\left[A_{1}, A_{2}\right]$. If we define, for $A$ and $B=\left[B_{1}, B_{2}\right]$ from $W$, the relation

$$
d(A, B)=\max \left\{\left|A_{1}-B_{1}\right|,\left|A_{2}-B_{2}\right|\right\},
$$

then $(W, d)$ is a complete metric space. Bede [2] defined on $E^{1}$ the Hausdorff metric

$$
D(u, v)=\sup _{\alpha \in[0,1]} d\left([u]_{\alpha},[v]_{\alpha}\right) .
$$

Lemma 1.4 (see [2]). Let $x, y, z, u \in E^{1}$ and $k \in \mathbb{R}$. Then 
(i) $\left(E^{1}, D\right)$ is a complete metric space;

(ii) $D(k x, k y)=|k| D(x, y)$;

(iii) $D(x+y, z+y)=D(x, z)$;

(iv) $D(x+y, z+u) \leq D(x, z)+D(y, u)$.

Matloka [15] introduced bounded and convergent sequences of fuzzy numbers and studied their properties. In [20], Pringsheim gave a notion of convergence of double sequences. Savaş [21] extended this notion of Pringsheim's convergence to double sequences of fuzzy numbers.

Definition 1.5 (see $[15,21]$ ). A double sequence $x=\left(x_{j k}\right)$ of fuzzy numbers is a function $x: \mathbb{N} \times \mathbb{N} \rightarrow E^{1}$. The fuzzy number $x_{j k}$ denotes the value of the function at a point $(j, k) \in \mathbb{N} \times \mathbb{N}$ and is called the $(j, k)$-th term of the double sequence $x$. By ${ }_{2} W^{F}$ we denote the set of all double sequences of fuzzy numbers.

Definition 1.6 (see [21]). Consider the sequence $\left(x_{j k}\right) \in{ }_{2} W^{F}$. If for every $\epsilon>0$ there exists $n_{0}=n_{0}(\epsilon) \in \mathbb{N}$ and $L \in E^{1}$ such that $D\left(x_{j k}, L\right)<\epsilon$ for all $j, k>n_{0}$, then we say that the sequence $\left(x_{j k}\right)$ converges in the sense of Pringsheim to the limit $L$ and write $P-\lim _{j, k} x_{j k}=L$.

Definition 1.7 (see [22]). A sequence $x=\left(x_{j k}\right) \in{ }_{2} W^{F}$ is said to be statistically convergent to $L \in E^{1}$ if, for every $\epsilon>0, \delta_{2}(K(m, n, \epsilon))=0$, where $K(m, n, \epsilon)=\left\{(j, k): j \leq m, k \leq n: D\left(x_{j k}, L\right) \geq \epsilon\right\}$. In this case we write $s t_{2}-\lim x_{j k}=L$.

Precisely, $s t_{2}-\lim x_{j k}=L$ if

$$
P-\lim _{m, n} \frac{1}{m n}\left|(j, k) ; j \leq m, k \leq n: D\left(x_{j k}, L\right) \geq \epsilon\right|=0 .
$$

\section{Main results and discussions}

Let $p:=\left(p_{j}\right)_{j=0}^{\infty}$ and $q:=\left(q_{j}\right)_{k=0}^{\infty}$ be sequences of non-negative numbers such that $p_{0}, q_{0}>0$,

$$
P_{m}:=\sum_{j=0}^{m} p_{m} \rightarrow \infty \text { as } m \rightarrow \infty,
$$

and

$$
Q_{n}:=\sum_{k=0}^{n} q_{n} \rightarrow \infty \text { as } n \rightarrow \infty .
$$

The weighted mean $t_{m n}$ of $x=\left(x_{j k}\right) \in{ }_{2} W^{F}$ is defined as

$$
t_{m n}:=\frac{1}{P_{m} Q_{n}} \sum_{j=0}^{m} \sum_{k=0}^{n} p_{j} q_{k} x_{j k}, \quad m, n \geq 0 .
$$


Definition 2.1. If $t_{m n}$ converges to $L$ as $\min (m, n) \rightarrow \infty$, then we say that the sequence $x=\left(x_{j k}\right) \in{ }_{2} W^{F}$ is $\left(\bar{N}^{2}, p, q\right)^{F}$-summable to $L$.

Definition 2.2. A sequence $x=\left(x_{j k}\right) \in{ }_{2} W^{F}$ is called $\left(\bar{N}^{2}, p, q\right)^{F_{-}}$ statistically summable to $L$ if $s t_{2^{-}} \lim t_{m n}=L$.

Definition 2.3. Let $K \subseteq \mathbb{N} \times \mathbb{N}$ and $\epsilon>0$. We define the double weighted $\epsilon$-density of $K$ by

$$
\delta_{\bar{N}_{2}}(K, \epsilon):=\lim _{m, n} \frac{1}{P_{m} Q_{n}}\left|K_{P_{m} Q_{n}}(\epsilon)\right|
$$

provided the limit exists, where

$$
K_{P_{m} Q_{n}}(\epsilon):=\left\{(j, k) ; j \leq P_{m}, k \leq Q_{n}: p_{j} q_{k} D\left(x_{j k}, L\right) \geq \epsilon\right\},
$$

$\liminf p_{j}>0, \liminf q_{j}>0$. A sequence $x=\left(x_{j k}\right) \in{ }_{2} W^{F}$ is said to be

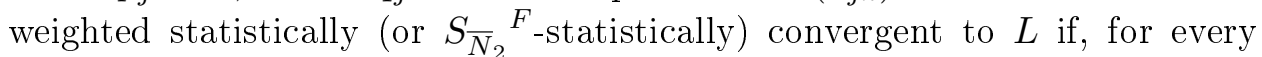
$\epsilon>0, \delta_{\bar{N}_{2}}(K, \epsilon)=0$.

Theorem 2.4. Let $p_{j} q_{k} D\left(x_{j k}, L\right) \leq M$ for all $j, k \in \mathbb{N}$. If a sequence $x=\left(x_{j k}\right) \in{ }_{2} W^{F}$ is $S_{\bar{N}_{2}}{ }^{F}$-statistically convergent to $L$, then it is $\left(\bar{N}^{2}, p, q\right)^{F_{-}}$ statistically summable to $L$.

Proof. We have

$$
\begin{aligned}
& D\left(t_{m n}, L\right)=D\left(\frac{1}{P_{m} Q_{n}} \sum_{j=0}^{m} \sum_{k=0}^{n} p_{j} q_{k} x_{j k}, L\right) \\
& \leq \frac{1}{P_{m} Q_{n}} \sum_{j=0}^{m} \sum_{k=0}^{n} p_{j} q_{k} D\left(x_{j k}, L\right) \\
& =\frac{1}{P_{m} Q_{n}} \sum_{\substack{j=0 \\
(j, k) \in K_{P_{m} Q_{n}}(\epsilon)}}^{m} \sum_{j=0}^{n} q_{k} D\left(x_{j k}, L\right)+\frac{1}{P_{m} Q_{n}} \sum_{\substack{j=0 k=0 \\
(j, k) \notin K_{P_{m} Q_{n}}(\epsilon)}}^{m} p_{j} q_{k} D\left(x_{j k}, L\right) \\
& \leq\left(\sup _{j, k} p_{j} q_{k} D\left(x_{j k}, L\right)\right)\left|K_{P_{m} Q_{n}}(\epsilon)\right|+\frac{1}{P_{m} Q_{n}} \sum_{j=0}^{m} \sum_{k=0}^{n} \epsilon \\
& (j, k) \notin K_{P_{m} Q_{n}}(\epsilon) \\
& \leq \frac{1}{P_{m} Q_{n}} M\left|K_{P_{m} Q_{n}}(\epsilon)\right|+\epsilon \rightarrow 0+\epsilon \text { as } m, n \rightarrow \infty .
\end{aligned}
$$

This implies that $t_{m n} \rightarrow L$.

Remark 2.5. If we set $p_{j}=1, q_{k}=1(j, k=0,1,2, \ldots)$ in Theorem 2.4 then we get Theorem 4.1 of [19].

It follows from Theorem 2.6 of [6] and Example 4.2 of [19] that the converse implication of Theorem 2.4 does not hold in general. We find some conditions 
under which the converse also holds. We give two-sided Tauberian conditions, each of which is necessary and sufficient for statistical convergence to be followed from statistical summability by weighted means.

Definition 2.6. We define the sums

$$
\tau_{m n}^{>}:=\frac{1}{\left(P_{\lambda_{m}}-P_{m}\right)\left(Q_{\lambda_{n}}-Q_{n}\right)} \sum_{j=m+1}^{\lambda_{m}} \sum_{k=n+1}^{\lambda_{n}} p_{j} q_{k} x_{j k} \quad(\lambda>1)
$$

and

$$
\tau_{m n}^{<}:=\frac{1}{\left(P_{m}-P_{\lambda_{m}}\right)\left(Q_{n}-Q_{\lambda_{n}}\right)} \sum_{j=\lambda_{m}+1}^{m} \sum_{k=\lambda_{n}+1}^{n} p_{j} q_{k} x_{j k} \quad(0<\lambda<1)
$$

for sufficiently large non-negative integers $m, n$. Here $\lambda_{n}$ denotes the integer part $[\lambda n]$ of the product $\lambda n$.

Fridy and Orhan [12] introduced the idea of statistical limit inferior and statistical limit superior of a sequence of real numbers. Móricz and Orhan [16] proved the following lemma.

Lemma 2.7 (see [16]). If $\left\{R_{m}\right\}$ is a non-decreasing sequence of positive numbers, then the conditions

$$
\text { st }-\liminf _{m \rightarrow \infty} \frac{R_{\lambda_{m}}}{R_{m}}>1 \text { for every } \lambda>1
$$

and

$$
\text { st }-\liminf _{m \rightarrow \infty} \frac{R_{m}}{R_{\lambda_{m}}}>1 \text { for every } 0<\lambda<1
$$

are equivalent.

Lemma 2.8. Let $p=\left(p_{j}\right)_{j=0}^{\infty}$ and $q=\left(q_{k}\right)_{k=0}^{\infty}$ be sequences of non-negative numbers such that $p_{0}, q_{0}>0$. If a sequence $x=\left(x_{m n}\right) \in{ }_{2} W^{F}$ is $\left(\bar{N}^{2}, p, q\right)^{F}$. statistically summable to $L$, then, for $\lambda>0$,

$$
s t_{2}-\lim t_{\lambda_{m} \lambda_{n}}=L, \quad s t_{2-} \lim t_{\lambda_{m} n}=L, \text { and } s t_{2}-\lim t_{m \lambda_{n}}=L .
$$

Proof. We only show $s t_{2^{-}} \lim t_{\lambda_{m} \lambda_{n}}=L$, the proofs of other cases are similar. For $\lambda=1$, it is trivial.

Suppose $\lambda>1$. For all $M, N \geq 1$ and $\epsilon>0$,

$$
\left\{m \leq M, n \leq N: D\left(t_{\lambda_{m} \lambda_{n}}, L\right) \geq \epsilon\right\} \subseteq\left\{m \leq \lambda_{m}, n \leq \lambda_{n}: D\left(t_{\lambda_{m} \lambda_{n}}, L\right) \geq \epsilon\right\},
$$

whence we find

$$
\begin{aligned}
& \frac{1}{M N}\left|\left\{m \leq M, n \leq N: D\left(t_{\lambda_{m} \lambda_{n}}, L\right) \geq \epsilon\right\}\right| \\
& \quad \leq \frac{\lambda . \lambda}{\lambda_{M} \lambda_{N}}\left|\left\{m \leq \lambda_{M}, n \leq \lambda_{N}: D\left(t_{m n}, L\right) \geq \epsilon\right\}\right| \rightarrow 0 \text { as } \min (M, N) \rightarrow \infty .
\end{aligned}
$$

The last step follows from the fact that $x$ is $\left(\bar{N}^{2}, p, q\right)^{F}$-statistically summable to $L$. 
For $0<\lambda<1$, the sequence $\left(\lambda_{j}\right)_{j=1}^{\infty}$ is non-decreasing. If integers $j$ and $s$ are such that $m=\lambda_{j}=\lambda_{j+1}=\cdots=\lambda_{j+s-1}<\lambda_{j+s}$, then

$$
m \leq \lambda j<\lambda(j+1)<\cdots<\lambda(j+s-1)<m+1 \leq \lambda(j+s) .
$$

This implies that

$$
m+\lambda(s-1) \leq \lambda j+\lambda(s-1)=\lambda(j+s-1)=m+1
$$

and so $s<1+\lambda^{-1}$. Here the pairs $(j, k)$ with $\lambda_{j}=m$ and $\lambda_{k}=n$ occur at most $\left(1+\lambda^{-1}\right)^{2}$ times. Moreover, we have $\lambda_{N} / N \leq 2 \lambda$ for $N \geq \max \left\{\lambda^{-1}-\right.$ $2,0\}$. Consequently,

$$
\begin{aligned}
& \frac{1}{M N}\left|\left\{m \leq M, n \leq N: D\left(t_{\lambda_{m} \lambda_{n}}, L\right) \geq \epsilon\right\}\right| \\
& \quad \leq \frac{\left(1+\lambda^{-1}\right)^{2}}{M N}\left|\left\{m \leq \lambda_{M}, n \leq \lambda_{N}: D\left(t_{m n}, L\right) \geq \epsilon\right\}\right| \\
& \quad \leq \frac{\left(1+\lambda^{-1}\right)^{2}(2 \lambda)^{2}}{\lambda_{M} \lambda_{N}}\left|\left\{m \leq \lambda_{M}, n \leq \lambda_{N}: D\left(t_{m n}, L\right) \geq \epsilon\right\}\right| \rightarrow 0
\end{aligned}
$$

as $\min (M, N) \rightarrow \infty$. This step completes the proof.

Lemma 2.9. Let the sequences $p$ and $q$ be the same as in Lemma 2.8.

(1) If $\lambda>1, \lambda_{m}>m$, and $\lambda_{n}>n$, then

$$
\begin{aligned}
D\left(\tau_{m n}^{>}, t_{m n}\right) \leq & \frac{P_{\lambda_{m}} Q_{\lambda_{n}}}{\left(P_{\lambda_{m}}-P_{m}\right)\left(Q_{\lambda_{n}}-Q_{n}\right)}\left(D\left(t_{\lambda_{m} \lambda_{n}}, t_{\lambda_{m} n}\right)+D\left(t_{m n}, t_{m \lambda_{n}}\right)\right) \\
& +\frac{P_{\lambda_{m}}}{P_{\lambda_{m}}-P_{m}} D\left(t_{\lambda_{m} n}, t_{m n}\right)+\frac{Q_{\lambda_{n}}}{Q_{\lambda_{n}}-Q_{n}} D\left(t_{m \lambda_{n}}, t_{m n}\right) .
\end{aligned}
$$

(2) If $0<\lambda<1, \lambda_{m}<m$, and $\lambda_{n}<n$, then

$$
\begin{aligned}
D\left(\tau_{m n}^{<}, t_{m n}\right) \leq & \frac{P_{\lambda_{m}} Q_{\lambda_{n}}}{\left(P_{m}-P_{\lambda_{m}}\right)\left(Q_{n}-Q_{\lambda_{n}}\right)}\left(D\left(t_{\lambda_{m} \lambda_{n}}, t_{\lambda_{m} n}\right)+D\left(t_{m n}, t_{m \lambda_{n}}\right)\right) \\
& +\frac{P_{\lambda_{m}}}{P_{m}-P_{\lambda_{m}}} D\left(t_{\lambda_{m} n}, t_{m n}\right)+\frac{Q_{\lambda_{n}}}{Q_{n}-Q_{\lambda_{n}}} D\left(t_{m \lambda_{n}}, t_{m n}\right) .
\end{aligned}
$$

Proof. (1) Let $\lambda>1, \lambda_{m}>m$, and $\lambda_{n}>n$. By definition of $\tau_{m n}^{>}$and relations (ii), (iv) of Lemma 1.4 we have

$$
\begin{aligned}
& D\left(\tau_{m n}^{>}, t_{m n}\right)=D\left(\frac{1}{\left(P_{\lambda_{m}}-P_{m}\right)\left(Q_{\lambda_{n}}-Q_{n}\right)} \sum_{j=m+1}^{\lambda_{m}} \sum_{k=n+1}^{\lambda_{n}} p_{j} q_{k} x_{j k}, t_{m n}\right) \\
& =D\left(\frac{1}{\left(P_{\lambda_{m}}-P_{m}\right)\left(Q_{\lambda_{n}}-Q_{n}\right)} \sum_{j=m+1}^{\lambda_{m}} \sum_{k=n+1}^{\lambda_{n}} p_{j} q_{k} x_{j k}\right. \\
& \quad+\frac{1}{\left(P_{\lambda_{m}}-P_{m}\right)\left(Q_{\lambda_{n}}-Q_{n}\right)}\left(\sum_{j=0}^{m} \sum_{k=n+1}^{\lambda_{n}}+\sum_{j=m+1}^{\lambda_{m}} \sum_{k=0}^{n}+2 \sum_{j=0}^{m} \sum_{k=0}^{n}\right) p_{j} q_{k} x_{j k}, \\
& \left.t_{m n}+\frac{1}{\left(P_{\lambda_{m}}-P_{m}\right)\left(Q_{\lambda_{n}}-Q_{n}\right)}\left(\sum_{j=0}^{m} \sum_{k=n+1}^{\lambda_{n}}+\sum_{j=m+1}^{\lambda_{m}} \sum_{k=0}^{n}+2 \sum_{j=0}^{m} \sum_{k=0}^{n}\right) p_{j} q_{k} x_{j k}\right)
\end{aligned}
$$




$$
\begin{aligned}
& =D\left(\frac{1}{\left(P_{\lambda_{m}}-P_{m}\right)\left(Q_{\lambda_{n}}-Q_{n}\right)} \sum_{j=0}^{\lambda_{m}} \sum_{k=0}^{\lambda_{n}} p_{j} q_{k} x_{j k}\right. \\
& +\frac{1}{\left(P_{\lambda_{m}}-P_{m}\right)\left(Q_{\lambda_{n}}-Q_{n}\right)} \sum_{j=0}^{m} \sum_{k=0}^{n} p_{j} q_{k} x_{j k}, \\
& t_{m n}+\frac{1}{\left(P_{\lambda_{m}}-P_{m}\right)\left(Q_{\lambda_{n}}-Q_{n}\right)} \sum_{j=0}^{m} \sum_{k=0}^{\lambda_{n}} p_{j} q_{k} x_{j k} \\
& \left.+\frac{1}{\left(P_{\lambda_{m}}-P_{m}\right)\left(Q_{\lambda_{n}}-Q_{n}\right)} \sum_{j=0}^{\lambda_{m}} \sum_{k=0}^{n} p_{j} q_{k} x_{j k}\right) \\
& =D\left(\frac{P_{\lambda_{m}} Q_{\lambda_{n}}}{\left(P_{\lambda_{m}}-P_{m}\right)\left(Q_{\lambda_{n}}-Q_{n}\right)} t_{\lambda_{m} \lambda_{n}}+\frac{P_{m} Q_{n}}{\left(P_{\lambda_{m}}-P_{m}\right)\left(Q_{\lambda_{n}}-Q_{n}\right)} t_{m n}\right. \\
& \left.t_{m n}+\frac{P_{\lambda_{m}} Q_{n}}{\left(P_{\lambda_{m}}-P_{m}\right)\left(Q_{\lambda_{n}}-Q_{n}\right)} t_{\lambda_{m} n}+\frac{P_{m} Q_{\lambda_{n}}}{\left(P_{\lambda_{m}}-P_{m}\right)\left(Q_{\lambda_{n}}-Q_{n}\right)} t_{m \lambda_{n}}\right) \\
& =D\left(\frac{P_{\lambda_{m}} Q_{\lambda_{n}}}{\left(P_{\lambda_{m}}-P_{m}\right)\left(Q_{\lambda_{n}}-Q_{n}\right)} t_{\lambda_{m} \lambda_{n}}+\frac{P_{m} Q_{n}}{\left(P_{\lambda_{m}}-P_{m}\right)\left(Q_{\lambda_{n}}-Q_{n}\right)} t_{m n}\right. \\
& +\frac{P_{\lambda_{m}}}{\left(P_{\lambda_{m}}-P_{m}\right)} t_{\lambda_{m} n}+\frac{Q_{\lambda_{n}}}{\left(Q_{\lambda_{n}}-Q_{n}\right)} t_{m \lambda_{n}}, t_{m n}+\frac{P_{\lambda_{m}} Q_{n}}{\left(P_{\lambda_{m}}-P_{m}\right)\left(Q_{\lambda_{n}}-Q_{n}\right)} t_{\lambda_{m} n} \\
& \left.+\frac{P_{\lambda_{m}}}{\left(P_{\lambda_{m}}-P_{m}\right)} t_{\lambda_{m} n}+\frac{P_{m} Q_{\lambda_{n}}}{\left(P_{\lambda_{m}}-P_{m}\right)\left(Q_{\lambda_{n}}-Q_{n}\right)} t_{m \lambda_{n}}+\frac{Q_{\lambda_{n}}}{\left(Q_{\lambda_{n}}-Q_{n}\right)} t_{m \lambda_{n}}\right) \\
& =D\left(\frac{P_{\lambda_{m}} Q_{\lambda_{n}}}{\left(P_{\lambda_{m}}-P_{m}\right)\left(Q_{\lambda_{n}}-Q_{n}\right)} t_{\lambda_{m} \lambda_{n}}+\frac{P_{m} Q_{n}}{\left(P_{\lambda_{m}}-P_{m}\right)\left(Q_{\lambda_{n}}-Q_{n}\right)} t_{m n}+\frac{P_{\lambda_{m}}}{\left(P_{\lambda_{m}}-P_{m}\right)} t_{\lambda_{m} n}\right. \\
& \left.+\frac{Q_{\lambda_{n}}}{\left(Q_{\lambda_{n}}-Q_{n}\right)} t_{m \lambda_{n}}, t_{m n}+\frac{P_{\lambda_{m}} Q_{\lambda_{n}}}{\left(P_{\lambda_{m}}-P_{m}\right)\left(Q_{\lambda_{n}}-Q_{n}\right)}\left(t_{\lambda_{m} n}+t_{m \lambda_{n}}\right)\right) \\
& \leq \frac{P_{\lambda_{m}} Q_{\lambda_{n}}}{\left(P_{\lambda_{m}}-P_{m}\right)\left(Q_{\lambda_{n}}-Q_{n}\right)} D\left(t_{\lambda_{m} \lambda_{n}}, t_{\lambda_{m} n}\right)+D\left(\frac{P_{m} Q_{n}}{\left(P_{\lambda_{m}}-P_{m}\right)\left(Q_{\lambda_{n}}-Q_{n}\right)} t_{m n}\right. \\
& +\frac{P_{\lambda_{m}}}{\left(P_{\lambda_{m}}-P_{m}\right)} t_{\lambda_{m} n}+\frac{Q_{\lambda_{n}}}{\left(Q_{\lambda_{n}}-Q_{n}\right)} t_{m \lambda_{n}}+\frac{P_{m}}{\left(P_{\lambda_{n}}-P_{n}\right)} t_{m n}+\frac{Q_{n}}{\left(Q_{\lambda_{n}}-Q_{n}\right)} t_{m n}+t_{m n}, \\
& \left.t_{m n}+\frac{P_{\lambda_{m}} Q_{\lambda_{n}}}{\left(P_{\lambda_{m}}-P_{m}\right)\left(Q_{\lambda_{n}}-Q_{n}\right)} t_{m \lambda_{n}}+\frac{P_{m}}{\left(P_{\lambda_{n}}-P_{n}\right)} t_{m n}+\frac{Q_{n}}{\left(Q_{\lambda_{n}}-Q_{n}\right)} t_{m n}+t_{m n}\right) \\
& =\frac{P_{\lambda_{m}} Q_{\lambda_{n}}}{\left(P_{\lambda_{m}}-P_{m}\right)\left(Q_{\lambda_{n}}-Q_{n}\right)} D\left(t_{\lambda_{m} \lambda_{n}}, t_{\lambda_{m} n}\right)+D\left(\frac{P_{\lambda_{m}} Q_{\lambda_{n}}}{\left(P_{\lambda_{m}}-P_{m}\right)\left(Q_{\lambda_{n}}-Q_{n}\right)} t_{m n}\right. \\
& +\frac{P_{\lambda_{m}}}{\left(P_{\lambda_{m}}-P_{m}\right)} t_{\lambda_{m} n}+\frac{Q_{\lambda_{n}}}{\left(Q_{\lambda_{n}}-Q_{n}\right)} t_{m \lambda_{n}} \\
& \left.\frac{P_{\lambda_{m}} Q_{\lambda_{n}}}{\left(P_{\lambda_{m}}-P_{m}\right)\left(Q_{\lambda_{n}}-Q_{n}\right)} t_{m \lambda_{n}}+\frac{P_{\lambda_{m}}}{\left(P_{\lambda_{n}}-P_{n}\right)} t_{m n}+\frac{Q_{\lambda_{n}}}{\left(Q_{\lambda_{n}}-Q_{n}\right)} t_{m n}\right) \\
& \leq \frac{P_{\lambda_{m}} Q_{\lambda_{n}}}{\left(P_{\lambda_{m}}-P_{m}\right)\left(Q_{\lambda_{n}}-Q_{n}\right)}\left(D\left(t_{\lambda_{m} \lambda_{n}}, t_{\lambda_{m} n}\right)+D\left(t_{m n}, t_{m \lambda_{n}}\right)\right) \\
& +\frac{P_{\lambda_{m}}}{\left(P_{\lambda_{m}}-P_{m}\right)} D\left(t_{\lambda_{m} n}, t_{m n}\right)+\frac{Q_{\lambda_{n}}}{\left(Q_{\lambda_{n}}-Q_{n}\right)} D\left(t_{m \lambda_{n}}, t_{m n}\right) \text {. }
\end{aligned}
$$


(2) Let $0<\lambda<1, \lambda_{m}<m$ and $\lambda_{n}<n$. By definition of $\tau_{m n}^{<}$and relations (ii), (iv) of Lemma 1.4 we have

$$
\begin{aligned}
& D\left(\tau_{m n}^{<}, t_{m n}\right)=D\left(\frac{1}{\left(P_{m}-P_{\lambda_{m}}\right)\left(Q_{n}-Q_{\lambda_{n}}\right)} \sum_{j=\lambda_{m}+1}^{m} \sum_{k=\lambda_{n}+1}^{n} p_{j} q_{k} x_{j k}, t_{m n}\right) \\
& =D\left(\frac{1}{\left(P_{m}-P_{\lambda_{m}}\right)\left(Q_{n}-Q_{\lambda_{n}}\right)} \sum_{j=\lambda_{m}+1}^{m} \sum_{k=\lambda_{n}+1}^{n} p_{j} q_{k} x_{j k}\right. \\
& +\frac{1}{\left(P_{m}-P_{\lambda_{m}}\right)\left(Q_{n}-Q_{\lambda_{n}}\right)}\left(\sum_{j=0}^{\lambda_{m}} \sum_{k=\lambda_{n}+1}^{n}+\sum_{j=\lambda_{m}+1}^{m} \sum_{k=0}^{\lambda_{n}}+2 \sum_{j=0}^{\lambda_{m}} \sum_{k=0}^{\lambda_{n}}\right) p_{j} q_{k} x_{j k}, \\
& \left.t_{m n}+\frac{1}{\left(P_{m}-P_{\lambda_{m}}\right)\left(Q_{n}-Q_{\lambda_{n}}\right)}\left(\sum_{j=0}^{\lambda_{m}} \sum_{k=\lambda_{n}+1}^{n}+\sum_{j=\lambda_{m}+1}^{m} \sum_{k=0}^{\lambda_{n}}+2 \sum_{j=0}^{\lambda_{m}} \sum_{k=0}^{\lambda_{n}}\right) p_{j} q_{k} x_{j k}\right) \\
& =D\left(\frac{1}{\left(P_{m}-P_{\lambda_{m}}\right)\left(Q_{n}-Q_{\lambda_{n}}\right)} \sum_{j=0}^{\lambda_{m}} \sum_{k=0}^{\lambda_{n}} p_{j} q_{k} x_{j k}\right. \\
& +\frac{1}{\left(P_{m}-P_{\lambda_{m}}\right)\left(Q_{n}-Q_{\lambda_{n}}\right)} \sum_{j=0}^{m} \sum_{k=0}^{n} p_{j} q_{k} x_{j k}, \\
& t_{m n}+\frac{1}{\left(P_{m}-P_{\lambda_{m}}\right)\left(Q_{n}-Q_{\lambda_{n}}\right)} \sum_{j=0}^{m} \sum_{k=0}^{\lambda_{n}} p_{j} q_{k} x_{j k} \\
& \left.+\frac{1}{\left(P_{m}-P_{\lambda_{m}}\right)\left(Q_{n}-Q_{\lambda_{n}}\right)} \sum_{j=0}^{\lambda_{m}} \sum_{k=0}^{n} p_{j} q_{k} x_{j k}\right) \\
& =D\left(\frac{P_{\lambda_{m}} Q_{\lambda_{n}}}{\left(P_{m}-P_{\lambda_{m}}\right)\left(Q_{n}-Q_{\lambda_{n}}\right)} t_{\lambda_{m} \lambda_{n}}+\frac{P_{m} Q_{n}}{\left(P_{m}-P_{\lambda_{m}}\right)\left(Q_{n}-Q_{\lambda_{n}}\right)} t_{m n},\right. \\
& \left.t_{m n}+\frac{P_{\lambda_{m}} Q_{n}}{\left(P_{m}-P_{\lambda_{m}}\right)\left(Q_{n}-Q_{\lambda_{n}}\right)} t_{\lambda_{m} n}+\frac{P_{m} Q_{\lambda_{n}}}{\left(P_{m}-P_{\lambda_{m}}\right)\left(Q_{n}-Q_{\lambda_{n}}\right)} t_{m \lambda_{n}}\right) \\
& =D\left(\frac{P_{\lambda_{m}} Q_{\lambda_{n}}}{\left(P_{m}-P_{\lambda_{m}}\right)\left(Q_{n}-Q_{\lambda_{n}}\right)} t_{\lambda_{m} \lambda_{n}}+\frac{P_{m} Q_{n}}{\left(P_{m}-P_{\lambda_{m}}\right)\left(Q_{n}-Q_{\lambda_{n}}\right)} t_{m n},\right. \\
& t_{m n}+\frac{P_{\lambda_{m}} Q_{\lambda_{n}}}{\left(P_{m}-P_{\lambda_{m}}\right)\left(Q_{n}-Q_{\lambda_{n}}\right)} t_{\lambda_{m} n}+\frac{P_{\lambda_{m}} Q_{\lambda_{n}}}{\left(P_{m}-P_{\lambda_{m}}\right)\left(Q_{n}-Q_{\lambda_{n}}\right)} t_{m \lambda_{n}} \\
& \left.+\frac{P_{\lambda_{m}}}{\left(P_{m}-P_{\lambda_{m}}\right)} t_{\lambda_{m} n}+\frac{Q_{\lambda_{n}}}{\left(Q_{n}-Q_{\lambda_{n}}\right)} t_{m \lambda_{n}}\right) \\
& \leq \frac{P_{\lambda_{m}} Q_{\lambda_{n}}}{\left(P_{m}-P_{\lambda_{m}}\right)\left(Q_{n}-Q_{\lambda_{n}}\right)} D\left(t_{\lambda_{m} \lambda_{n}}, t_{\lambda_{m} n}\right)+D\left(\frac{P_{m} Q_{n}}{\left(P_{m}-P_{\lambda_{m}}\right)\left(Q_{n}-Q_{\lambda_{n}}\right)} t_{m n},\right. \\
& \left.t_{m n}+\frac{P_{\lambda_{m}} Q_{\lambda_{n}}}{\left(P_{m}-P_{\lambda_{m}}\right)\left(Q_{n}-Q_{\lambda_{n}}\right)} t_{m \lambda_{n}}+\frac{P_{\lambda_{m}}}{\left(P_{m}-P_{\lambda_{m}}\right)} t_{\lambda_{m} n}+\frac{Q_{\lambda_{n}}}{\left(Q_{n}-Q_{\lambda_{n}}\right)} t_{m \lambda_{n}}\right) \\
& =\frac{P_{\lambda_{m}} Q_{\lambda_{n}}}{\left(P_{m}-P_{\lambda_{m}}\right)\left(Q_{n}-Q_{\lambda_{n}}\right)} D\left(t_{\lambda_{m} \lambda_{n}}, t_{\lambda_{m} n}\right)+D\left(\frac{P_{\lambda_{m}} Q_{\lambda_{n}}}{\left(P_{m}-P_{\lambda_{m}}\right)\left(Q_{n}-Q_{\lambda_{n}}\right)} t_{m n}\right.
\end{aligned}
$$




$$
\begin{aligned}
& \quad+\frac{P_{\lambda_{m}}}{\left(P_{m}-P_{\lambda_{m}}\right)} t_{m n}+\frac{Q_{\lambda_{n}}}{\left(Q_{n}-Q_{\lambda_{n}}\right)} t_{m n}+t_{m n}, t_{m n}+\frac{P_{\lambda_{m}} Q_{\lambda_{n}}}{\left(P_{m}-P_{\lambda_{m}}\right)\left(Q_{n}-Q_{\lambda_{n}}\right)} t_{m \lambda_{n}} \\
& \left.\quad+\frac{P_{\lambda_{m}}}{\left(P_{m}-P_{\lambda_{m}}\right)} t_{\lambda_{m} n}+\frac{Q_{\lambda_{n}}}{\left(Q_{n}-Q_{\lambda_{n}}\right)} t_{m \lambda_{n}}\right) \\
& \leq \frac{P_{\lambda_{m} Q_{\lambda_{n}}}}{\left(P_{m}-P_{\lambda_{m}}\right)\left(Q_{n}-Q_{\lambda_{n}}\right)} D\left(t_{\lambda_{m} \lambda_{n}}, t_{\lambda_{m} n}\right)+\frac{P_{\lambda_{m} Q_{\lambda_{n}}}}{\left(P_{m}-P_{\lambda_{m}}\right)\left(Q_{n}-Q_{\lambda_{n}}\right)} D\left(t_{m n}, t_{m \lambda_{n}}\right) \\
& \quad+\frac{P_{\lambda_{m}}}{\left(P_{m}-P_{\lambda_{m}}\right)} D\left(t_{m n}, t_{\lambda_{m} n}\right)+\frac{Q_{\lambda_{n}}}{\left(Q_{n}-Q_{\lambda_{n}}\right)} D\left(t_{m n}, t_{m \lambda_{n}}\right) .
\end{aligned}
$$

Lemma is proved.

Theorem 2.10. Let $p=\left(p_{j}\right)_{j=0}^{\infty}$ and $q=\left(q_{k}\right)_{k=0}^{\infty}$ be sequences of nonnegative numbers such that $p_{0}, q_{0}>0$. Suppose that both sequences $\left(P_{m}\right)$ and $\left(Q_{n}\right)$ satisfy either (1) or (2). If a sequence $x=\left(x_{m n}\right) \in{ }_{2} W^{F}$ is $\left(\bar{N}^{2}, p, q\right)^{F}$-statistically summable to L, then, for $\lambda>1, s t_{2-} \lim _{m, n \rightarrow \infty} \tau_{m n}^{>}=L$, i.e.,

$$
s t_{2}{ }^{-} \lim _{m, n \rightarrow \infty} \frac{1}{\left(P_{\lambda_{m}}-P_{m}\right)\left(Q_{\lambda_{n}}-Q_{n}\right)} \sum_{j=m+1}^{\lambda_{m}} \sum_{k=n+1}^{\lambda_{n}} p_{j} q_{k} x_{j k}=L,
$$

and, for $0<\lambda<1$, st $2_{2-}^{-} \lim _{m, n \rightarrow \infty} \tau_{m n}^{<}=L$, i.e.,

$$
s t_{2-} \lim _{m, n \rightarrow \infty} \frac{1}{\left(P_{m}-P_{\lambda_{m}}\right)\left(Q_{n}-Q_{\lambda_{n}}\right)} \sum_{j=\lambda_{m}+1}^{m} \sum_{k=\lambda_{n}+1}^{n} p_{j} q_{k} x_{j k}=L .
$$

Proof. Suppose $\lambda>1$. If $P_{\lambda_{m}}>P_{m}$ and $Q_{\lambda_{n}}>Q_{n}$, then

$$
D\left(\tau_{m n}^{>}, L\right)=D\left(\tau_{m n}^{>}+t_{m} n, t_{m n}+L\right) \leq D\left(\tau_{m n}^{>}, t_{m n}\right)+D\left(t_{m n}, L\right),
$$

and using (3), we get

$$
\begin{aligned}
D\left(\tau_{m n}^{>}, L\right) \leq & \frac{P_{\lambda_{m}} Q_{\lambda_{n}}}{\left(P_{\lambda_{m}}-P_{m}\right)\left(Q_{\lambda_{n}}-Q_{n}\right)} D\left(t_{\lambda_{m} \lambda_{n}}, t_{\lambda_{m} n}\right) \\
& +\frac{P_{\lambda_{m}} Q_{\lambda_{n}}}{\left(P_{\lambda_{m}}-P_{m}\right)\left(Q_{\lambda_{n}}-Q_{n}\right)} D\left(t_{m n}, t_{m \lambda_{n}}\right)-\frac{P_{\lambda_{m}}}{P_{\lambda_{m}}-P_{m}} D\left(t_{\lambda_{m} n}, t_{m n}\right) \\
& -\frac{Q_{\lambda_{n}}}{Q_{\lambda_{n}}-Q_{n}} D\left(t_{m \lambda_{n}}, t_{m n}\right)+D\left(t_{m n}, L\right) .
\end{aligned}
$$

By (1) we obtain

$$
\begin{aligned}
& \text { st- } \limsup _{m \rightarrow \infty} \frac{P_{\lambda_{m}}}{P_{\lambda_{m}}-P_{m}}=\left\{s t-\liminf _{m \rightarrow \infty}\left(1-\frac{P_{m}}{P_{\lambda_{m}}}\right)\right\}^{-1} \\
& \quad=\left\{1-\left(s t-\limsup _{m \rightarrow \infty} \frac{P_{m}}{P_{\lambda_{m}}}\right)\right\}^{-1}=\left\{1-\frac{1}{s t-\liminf _{m \rightarrow \infty} \frac{P_{\lambda_{m}}}{P_{m}}}\right\}^{-1}<\infty .
\end{aligned}
$$

Similarly we have

$$
\text { st- } \limsup _{m \rightarrow \infty} \frac{Q_{\lambda_{n}}}{Q_{\lambda_{n}}-Q_{n}}<\infty .
$$

Thus, for any $\epsilon>0$, we conclude that

$$
\left\{m \leq M, n \leq N: D\left(\tau_{m n}^{>}, L\right) \geq \epsilon\right\}
$$




$$
\begin{aligned}
& \subseteq\left\{m \leq M, n \leq N: \frac{P_{\lambda_{m}} Q_{\lambda_{n}}}{\left(P_{\lambda_{m}}-P_{m}\right)\left(Q_{\lambda_{n}}-Q_{n}\right)} D\left(t_{\lambda_{m} \lambda_{n}}, t_{\lambda_{m} n}\right) \geq \frac{\epsilon}{5}\right\} \\
& \bigcup\left\{m \leq M, n \leq N: \frac{P_{\lambda_{m}} Q_{\lambda_{n}}}{\left(P_{\lambda_{m}}-P_{m}\right)\left(Q_{\lambda_{n}}-Q_{n}\right)} D\left(t_{m n}, t_{m \lambda_{n}}\right) \geq \frac{\epsilon}{5}\right\} \\
& \bigcup\left\{m \leq M, n \leq N: \frac{P_{\lambda_{m}}}{P_{\lambda_{m}}-P_{m}} D\left(t_{\lambda_{m} n}, t_{m n}\right) \geq \frac{\epsilon}{5}\right\} \\
& \bigcup\left\{m \leq M, n \leq N: \frac{Q_{\lambda_{n}}}{Q_{\lambda_{n}}-Q_{n}} D\left(t_{m \lambda_{n}}, t_{m n}\right) \geq \frac{\epsilon}{5}\right\} \\
&\left.\bigcup\left\{m \leq M, n \leq N: D\left(t_{m n}, L\right) \geq \frac{\epsilon}{5}\right)\right\} .
\end{aligned}
$$

Finally, using Lemma 2.8 and the fact that $x=\left(x_{m n}\right)$ is $\left(\bar{N}^{2}, p, q, 1,1\right)^{F_{-}}$ statistically summable to $L$, we get (5).

If $0<\lambda<1, P_{\lambda_{m}}<P_{m}, Q_{\lambda_{n}}>Q_{n}$, then, using the inequality (4) and proceeding in a similar manner as above, we obtain (6).

Theorem 2.11. Let the sequences $p, q$, and $\left(P_{m}\right),\left(Q_{n}\right)$ be the same as in Theorem 2.10. If a double sequence of fuzzy numbers $x=\left(x_{m n}\right)$ is $\left(\bar{N}^{2}, p, q\right)^{F}$-statistically summable to L, then $x$ is statistically convergent to L, i.e.,

$$
s t_{2}-\lim x_{m n}=L,
$$

if and only if one of the following statements hold for every $\epsilon>0$ :

$$
\begin{aligned}
& \inf _{\lambda>1} \limsup _{M, N \rightarrow \infty} \frac{1}{M N} \mid\{m \leq M, n \leq N: \\
& \left.\quad D\left(\frac{1}{\left(P_{\lambda_{m}}-P_{m}\right)\left(Q_{\lambda_{n}}-Q_{n}\right)} \sum_{j=m+1}^{\lambda_{m}} \sum_{k=n+1}^{\lambda_{n}} p_{j} q_{k} x_{j k}, x_{m n}\right) \geq \epsilon\right\} \mid=0
\end{aligned}
$$

or

$$
\begin{aligned}
& \quad \inf _{0<\lambda<1} \limsup _{M, N \rightarrow \infty} \frac{1}{M N} \mid\{m \leq M, n \leq N: \\
& \left.\quad D\left(\frac{1}{\left(P_{m}-P_{\lambda_{m}}\right)\left(Q_{n}-Q_{\lambda_{n}}\right)} \sum_{j=\lambda_{m}+1}^{m} \sum_{k=\lambda_{n}+1}^{n} p_{j} q_{k} x_{j k}, x_{m n}\right) \geq \epsilon\right\} \mid=0 .
\end{aligned}
$$

Proof. Let $x=\left(x_{m n}\right)$ be a double sequence of fuzzy numbers which is $\left(\bar{N}^{2}, p, q\right)^{F}$-statistically summable to $L$.

Necessity. Suppose that $x$ is statistically convergent to $L$. Then, using relations (5) and (7), we get (8). Similarly, (9) follows from (6) and (7).

Sufficiency. Suppose that one of the conditions (8) and (9) holds. To prove $s t_{2^{-}} \lim _{m, n \rightarrow \infty} x_{m n}=L$, it is enough to prove that

$$
s t_{2^{-}} \lim D\left(x_{m n}, t_{m n}\right)=0 .
$$


First, we suppose that (8) holds. Since in the case $\lambda>1$

$$
\begin{aligned}
& D\left(t_{m n}, x_{m n}\right) \leq D\left(t_{m n}, \tau_{m n}^{>}\right)+D\left(\tau_{m n}^{>}, x_{m n}\right) \\
& \leq \frac{P_{\lambda_{m}} Q_{\lambda_{n}}}{\left(P_{\lambda_{m}}-P_{m}\right)\left(Q_{\lambda_{n}}-Q_{n}\right)} D\left(t_{\lambda_{m} \lambda_{n}}, t_{\lambda_{m} n}\right)+\frac{P_{\lambda_{m}} Q_{\lambda_{n}}}{\left(P_{\lambda_{m}}-P_{m}\right)\left(Q_{\lambda_{n}}-Q_{n}\right)} D\left(t_{m n}, t_{m \lambda_{n}}\right) \\
& \quad+\frac{P_{\lambda_{m}}}{\left(P_{\lambda_{m}}-P_{m}\right)} D\left(t_{\lambda_{m} n}, t_{m n}\right)+\frac{Q_{\lambda_{n}}}{\left(Q_{\lambda_{n}}-Q_{n}\right)} D\left(t_{m \lambda_{n}}, t_{m n}\right) \\
& \quad+D\left(\frac{1}{\left(P_{\lambda_{m}}-P_{m}\right)\left(Q_{\lambda_{n}}-Q_{n}\right)} \sum_{j=m+1}^{\lambda_{m}} \sum_{k=n+1}^{\lambda_{n}} p_{j} q_{k} x_{j k}, x_{m n}\right),
\end{aligned}
$$

for any $\epsilon>0$ we have

$$
\begin{aligned}
& \left\{m \leq M, n \leq N: D\left(t_{m n}, x_{m n}\right) \geq \epsilon\right\} \\
& \subseteq\left\{m \leq M, n \leq N: \frac{P_{\lambda_{m}} Q_{\lambda_{n}}}{\left(P_{\lambda_{m}}-P_{m}\right)\left(Q_{\lambda_{n}}-Q_{n}\right)} D\left(t_{\lambda_{m} \lambda_{n}}, t_{\lambda_{m} n}\right) \geq \frac{\epsilon}{5}\right\} \\
& \quad \cup\left\{m \leq M, n \leq N: \frac{P_{\lambda_{m}} Q_{\lambda_{n}}}{\left(P_{\lambda_{m}}-P_{m}\right)\left(Q_{\lambda_{n}}-Q_{n}\right)} D\left(t_{m n}, t_{m \lambda_{n}}\right) \geq \frac{\epsilon}{5}\right\} \\
& \quad \cup\left\{m \leq M, n \leq N: \frac{P_{\lambda_{m}}}{\left(P_{\lambda_{m}}-P_{m}\right)} D\left(t_{\lambda_{m} n}, t_{m n}\right) \geq \frac{\epsilon}{5}\right\} \\
& \quad \cup\left\{m \leq M, n \leq N: \frac{Q_{\lambda_{n}}}{\left(Q_{\lambda_{n}}-Q_{n}\right)} D\left(t_{m \lambda_{n}}, t_{m n}\right) \geq \frac{\epsilon}{5}\right\} \\
& \quad \cup\left\{m \leq M, n \leq N: D\left(\frac{1}{\left(P_{\lambda_{m}}-P_{m}\right)\left(Q_{\lambda_{n}}-Q_{n}\right)} \sum_{j=m+1}^{\lambda_{m}} \sum_{k=n+1}^{\lambda_{n}} p_{j} q_{k} x_{j k}, x_{m n}\right) \geq \frac{\epsilon}{5}\right\} .
\end{aligned}
$$

By (8), for any given $\delta>0$, there exists $\lambda>1$ such that

$$
\begin{aligned}
& \limsup _{M, N \rightarrow \infty} \frac{1}{M N} \mid\{m \leq M, n \leq N: \\
& \left.\quad D\left(\frac{1}{\left(P_{\lambda_{m}}-P_{m}\right)\left(Q_{\lambda_{n}}-Q_{n}\right)} \sum_{j=m+1}^{\lambda_{m}} \sum_{k=n+1}^{\lambda_{n}} p_{j} q_{k} x_{j k}, x_{m n}\right) \geq \frac{\epsilon}{5}\right\} \mid \leq \delta .
\end{aligned}
$$

From Lemma 2.8 it follows that

$$
\begin{aligned}
& \limsup _{M, N \rightarrow \infty} \frac{1}{M N}\left|\left\{m \leq M, n \leq N: \frac{P_{\lambda_{m}} Q_{\lambda_{n}}}{\left(P_{\lambda_{m}}-P_{m}\right)\left(Q_{\lambda_{n}}-Q_{n}\right)} D\left(t_{\lambda_{m} \lambda_{n}}, t_{\lambda_{m} n}\right) \geq \frac{\epsilon}{5}\right\}\right|=0, \\
& \limsup _{M, N \rightarrow \infty} \frac{1}{M N}\left|\left\{m \leq M, n \leq N: \frac{P_{\lambda_{m}} Q_{\lambda_{n}}}{\left(P_{\lambda_{m}}-P_{m}\right)\left(Q_{\lambda_{n}}-Q_{n}\right)} D\left(t_{m n}, t_{m \lambda_{n}}\right) \geq \frac{\epsilon}{5}\right\}\right|=0, \\
& \limsup _{M, N \rightarrow \infty} \frac{1}{M N}\left|\left\{m \leq M, n \leq N: \frac{P_{\lambda_{m}}}{\left(P_{\lambda_{m}}-P_{m}\right)} D\left(t_{\lambda_{m} n}, t_{m n}\right) \geq \frac{\epsilon}{5}\right\}\right|=0, \\
& \limsup _{M, N \rightarrow \infty} \frac{1}{M N}\left|\left\{m \leq M, n \leq N: \frac{Q_{\lambda_{n}}}{\left(Q_{\lambda_{n}}-Q_{n}\right)} D\left(t_{m \lambda_{n}}, t_{m n}\right) \geq \frac{\epsilon}{5}\right\}\right|=0 .
\end{aligned}
$$

Thus we have

$$
\lim _{M, N \rightarrow \infty} \sup \frac{1}{M N}\left|\left\{m \leq M, n \leq N: D\left(x_{m n}, t_{m n}\right) \geq \epsilon\right\}\right| \leq \delta .
$$


Since $\delta$ is arbitrary, we can say that, for every $\epsilon>0$,

$$
\lim _{M, N \rightarrow \infty} \frac{1}{M N}\left|\left\{m \leq M, n \leq N: D\left(x_{m n}, t_{m n}\right) \geq \epsilon\right\}\right|=0,
$$

i.e., (10) is true.

Secondly, if (9) is satisfied, then (10) may be proved in a similar way, considering the case $0<\lambda<1$ with $P_{m}-P_{\lambda_{m}}$ and $Q_{n}-Q_{\lambda_{n}}$ instead of $P_{\lambda_{m}}-P_{m}$ and $Q_{\lambda_{n}}-Q_{n}$, respectively.

\section{Acknowledgements}

The authors benefited from the comments of learned referees. The authors also express their gratitude to University Grants Commission, New Delhi, India for offering fellowship to J. Gogoi via award letter no F./2015-16/NFO2015-17-OBC-ASS-36722/(SA-III/Website).

\section{References}

[1] Y. Altin, M. Mursaleen, and H. Altinok, Statistical summability $(C, 1)$ for sequences of fuzzy real numbers and a Tauberian theorem, J. Intell. Fuzzy Systems 21 (2010), 379-384.

[2] B. Bede, Mathematics of Fuzzy Sets and Fuzzy Logic, Springer, Heidelberg, 2013.

[3] N. L. Braha and M. Et, Tauberian theorems for Euler-Nörlund mean-convergent sequences of fuzzy numbers, Iran J. Fuzzy Syst. 14(2) (2017), 79-92.

[4] R. C. Buck, Generalized asymptotic density, Amer. J. Math. 75 (1953), 335-346.

[5] I. Çanak, Ü Totur, and Z. Önder, A Tauberian theorem for $(C, 1,1)$ summable double sequences of fuzzy numbers, Iran J. Fuzzy Syst. 14(1) (2017), 61-75.

[6] M. Cinar and M. Et, Generalized weighted statistical convergence of double sequences and applications, Filomat 30(3) (2016), 753-762.

[7] P. Diamond and P. Kloeden, Metric spaces of fuzzy sets, Fuzzy Sets and Systems 35 (1990), 241-249.

[8] D. Dubois and H. Prade, Operations on fuzzy numbers, Internat. J. Systems Sci. 9 (1978), 613-626.

[9] H. Dutta and J. Gogoi, Characterization of matrix classes involving some sets of summable double sequences of fuzzy numbers, J. Intell. Fuzzy Systems 34(6) (2018), 4279-4290.

[10] H. Dutta and J. Gogoi, Duals and matrix classes involving Cesàro type classes of sequences of fuzzy numbers, Adv. Fuzzy Syst. 2018, Art. ID 8636121, 9 pp.

[11] H. Fast, Sur la convergence statistique, Colloq. Math. 2 (1951), 241-244.

[12] J. A. Fridy and C. Orhan, Statistical limit superior and limit inferior, Proc. Amer. Math. Soc. 125 (1997), 3625-3631.

[13] R. Goetschel and W. Voxman, Elementary fuzzy calculus, Fuzzy Sets Syst. 18 (1986), 31-43.

[14] V. Loku, N. L. Braha, M. Et, and A. Tato, Tauberian theorems for generalized de la Vallée-Poussin mean-convergent sequences of fuzzy numbers, Bull. Math. Anal. Appl. $\mathbf{9}(2)$ (2017), 45-56.

[15] H. Matloka, Sequences of fuzzy numbers, BUSEFAL 28 (1986), 28-37.

[16] F. Móricz and C. Orhan, Tauberian conditions under which statistical convergence follows from statistical summability by weighted means, Studia Sci. Math. Hungar. 41 (2004), 391-403. 
[17] F. Móricz and B. E. Rhoades, Almost convergence of double sequences and strong regularity of summability matrices, Math. Proc. Cambridge Philos. Soc. 104 (1988), 283-294.

[18] F. Nuray and E. Savas, Statistical convergence of sequences of fuzzy numbers, Math. Slovaca 45(3) (1995), 269-273.

[19] Z. Önder, I. Çanak, and Ü. Totur, Tauberian theorems for statistically $(C, 1,1)$ summable double sequences of fuzzy numbers, Open Math. 15 (2017), 157-178.

[20] A. Pringsheim, Zur theorie der zweifach unendlichen Zahlenfolgen, Math. Ann. 53 (1900), 289-321.

[21] E. Savaş, A note on double sequences of fuzzy numbers, Turkish J. Math. 20 (1996), $175-178$.

[22] E. Savaş and M. Mursaleen, On statistically convergent double sequences of fuzzy numbers, Inform. Sci. 162 (2004), 183-192.

[23] I. J. Schoenberg, The integrability of certain functions and related summability methods, Amer. Math. Monthly 66 (1959), 361-375.

[24] P. V. Subrahmanyam, Cesàro summability for fuzzy real numbers, J. Anal. 7 (1999), $159-168$

[25] Ö. Talo and C. Bal, On statistical summability $(\bar{N}, P)$ of sequences of fuzzy numbers, Filomat 30(3) (2016), 873-884.

[26] E. Yavuz, Euler summability method of sequences of fuzzy numbers and a Tauberian theorem, J. Intell. Fuzzy Systems 32(1) (2017), 937-943.

[27] L. A. Zadeh, Fuzzy sets, Information and Control 8 (1965), 338-353.

[28] A. Zygmund, Trigonometric Series, Cambridge University Press, Cambridge, 1979.

Department of Mathematics, Gauhati University, Guwahati-781014, India

E-mail address: hemen_dutta08@rediffmail.com

E-mail address: jyotishmgogoi@gmail.com 